\title{
VALUE CHAIN SIMULATION IN AIRCRAFT PRODUCTION
}

\author{
Marvin Hermelijn \\ Jeroen Steenbakkers \\ Simon van der Weij \\ INCONTROL Simulation Solutions \\ Papendorpseweg 77 \\ Utrecht, 3528 BJ, THE NETHERLANDS
}

\begin{abstract}
Simulation has proven to be a successful method for analyzing and improving value chains and cost price analysis in complex environments, such as aircraft production. This case-study concerns the value chain simulator (VCS), developed by INCONTROL for Fokker Aerostructures. This VCS enables the users to deal with all relevant parameters for manufacturing, logistics and financing in early project phases.

The Fokker VCS is integrated in the current ERP system, so all relevant design parameters including Bill of Material, Bill of Processes and Bill of Resources are imported in the VCS and the simulation model is built up automatically. Since all financial parameters are included in the model, eventually the cost-price development of the manufactured parts over the full delivery period is calculated using simulation. The simulation application has already achieved benefits, but the final goal of this ongoing development is to accomplish an application which supports aircraft manufacturers in determining the Total Cost of Ownership for their products.
\end{abstract}

\section{INTRODUCTION}

This paper describes a simulation application for the modeling the value chain at the aircraft production company Fokker Aerostructures.

Fokker is a very well known brand in the aviation industry. Since almost a century the company has been involved in aviation industry as aircraft developer and manufacturer. Fokker Aerostructures is one of the business units and is specialized in the design, development and manufacturing of lightweight complex structures, modules and sub-assemblies. These are supplied to aircraft assembly and production facilities of many companies in the aerospace and defense industry.

Especially in the context of acquisition phases it is a big challenge for the company to determine cost prices or even the total cost of ownership for these products. The complexity of the processes, the extremely high quality standards in this industry, the high value of the parts and the long life-cycles are important factors in this challenge. But in an environment like aviation, the rapid sequence of innovations makes it even harder to determine costs. Innovation in techniques and materials, such as composites and sandwich materials (TAPAS 2011), are part of the daily routine in Fokker Research \& Development, and its results have to be implemented during the long-lasting production periods. As a result, during the contract terms there are changes in the costs of the value adding activities that are part of the value chain, creating uncertainties in cost price calculations.

To support the cost calculation methods and the decision making about the value chain for aircraft parts at Fokker Aerostructures, a simulation application has been developed by INCONTROL. This paper describes the developed application and its functionality. Section 2 gives a brief overview of the issues the company faces concerning their value chain, followed by a description of the conceptual design in 
Section 3. The structure of the simulation application is discussed in Section 4. Some first results are presented in Section 5 and finally, in chapter 6, some main conclusion and future work are discussed.

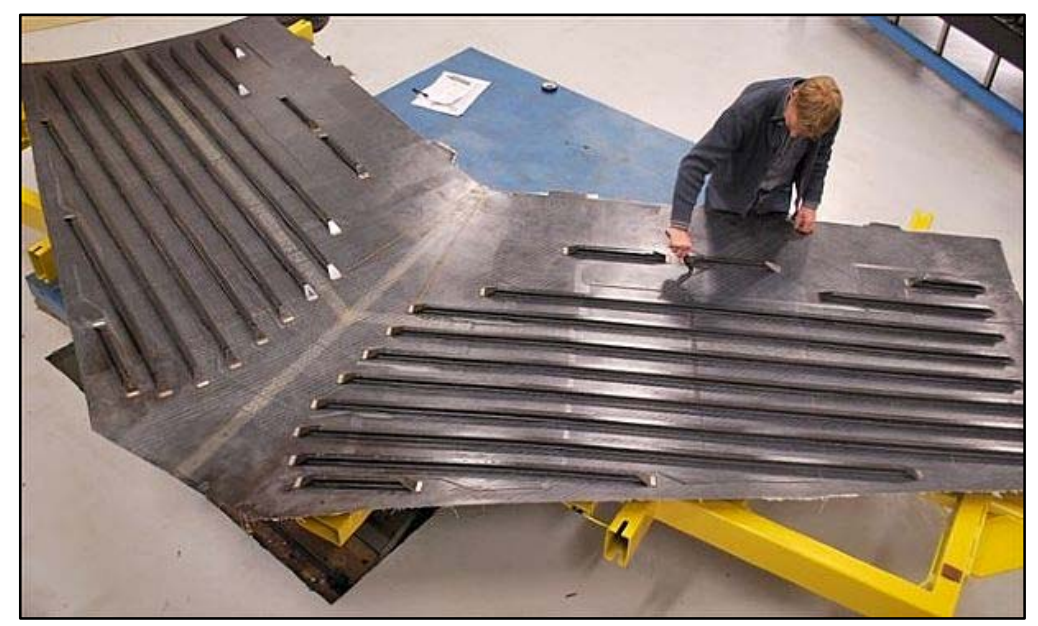

Figure 1: Example of innovative products

\section{VALUE CHAIN CHALLENGE}

Fokker supplies a wide range of parts and structures to its customers, using many different materials and processes. These products are often delivered during a timeframe of several to dozens of years. To be able to perform such programs rearrangement or investments in machinery and logistics is generally required. Therefore it is very important to be able to estimate the cost price of parts in the design phase, as this will also determine the proposed selling price for the supplied company and thus Fokker's competitiveness.

There are however many aspect that influence the cost price and the competitiveness. The value chain concept (Porter 1985) is the guideline to distinct and allocate costs of value adding activities. These include for instance, material costs, labor costs, capacity investments, but also less direct costs, such as inflation or learning curve effects. To define the total cost of a part, the following design stages are recognized:

1. Determine the product design: all necessary subparts, materials and their process steps are distinguished

2. Determine the production design: technical decisions regarding machinery and tooling are made.

3. Determine the logistics design: decisions regarding goods flow control and material handling are made.

4. Determine the supply chain design: decision regarding the supplier delivery quantities and moments are made.

Not just the cost of the value chains, but also external financial aspects need to be taken into account. For instance exchange rates, inflation or evolving costs for raw material or of great importance as the business is international and the programs have long delivery horizons.

In order to be able to deal with all uncertainties and properly estimate the costs of the designed parts over these planning horizons, Fokker Aerostructures requested the development of a value chain simulation application. 


\section{THE CONCEPT OF THE APPLICATION}

The first step in the development concerned the definition of the concept. During this conceptualization phase important stakeholders of all units in the design process and primary company activities were interviewed, including Industrial Engineers, Cost Estimators, Logistics Managers and Operations Managers. From these interviews all relevant aspects were derived and processed into an application design.

Some generally recognized requirement were the need for modularity and hierarchy of the application. A modular approach makes it possible to do an incremental development, which speeds up the date of commissioning for base modules, and enables the user to select or exclude (sub-)modules. The hierarchy of the structure means that different levels of detail can be defined, but a module cannot be used without its 'parent' module. The modular design of the application is depicted in Figure 2.

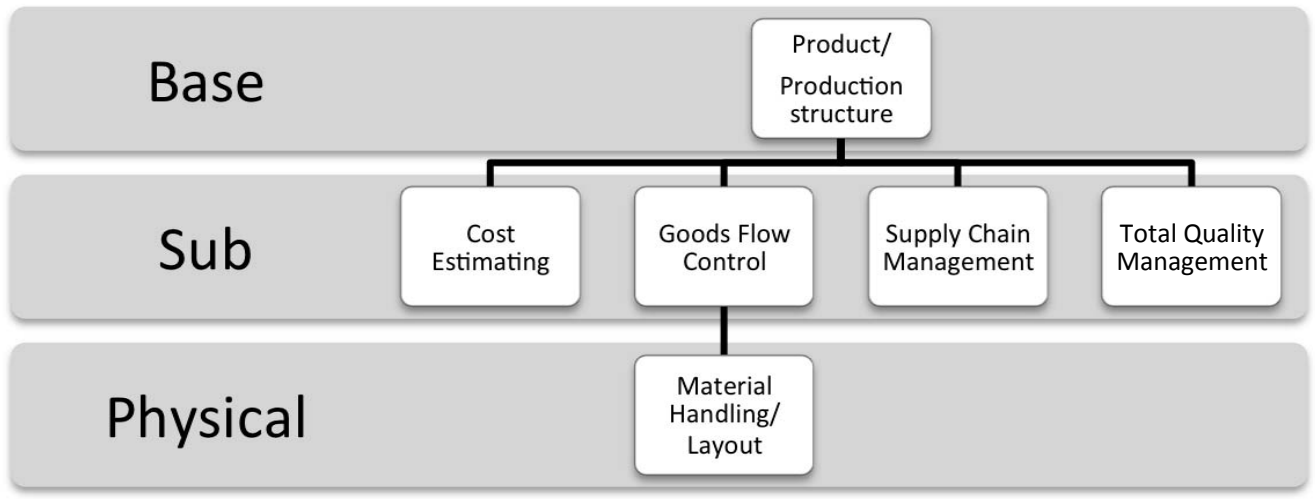

Figure 2: Modular design Fokker Value Chain Simulator

The base production module is always present, but the application had to be developed in such a way that several (or all) modules can be used together at the same time, depending on the user requirements. In each combination, only the corresponding settings and characteristics for selected sub models are available. If for example the Goods Flow Control module is used in combination with the Cost Estimating module, all relevant cost settings and cost output concerning Goods Flow Control are presented to the user.

The hierarchy requirements followed from the expressed wish to be able to use several levels of detail or even have some activities and processes represented by block-boxes with some simple stochastic parameters.

Another important requirement for the application concerned the way of using the tool. All conditions are there to use discrete event simulation: the complexity of the system, the dynamical time-dependent situation and the uncertainties, translated into stochastic distributions. As there are intended users for the application from several business units, including $R \& D$, sales and cost estimating, who do not have specific simulation knowledge and do not intend to become simulation specialists, the application needs to be very straightforward in use and the complex business information must be entered as system input by simple means. The solution had to found in a user-friendly interface, an automated connection to a generic external database and smart simulation objects, which contain all necessary functionalities and can be used for automated model set-up.

\subsection{Modules}

In order to create an overview of the modules that have so far been elaborated, the modules and their functionality, complexity and parameters are addressed in the following sections. 


\section{Hermelijn. Steenbakkers and Van Der Weij}

\subsubsection{Module Functionalities}

The core of the application is formed by the Base module, describing the product and production structure. The main purpose of this module is to create insight in the use of resource capacity, tools and other materials when producing a predefined set of orders. The starting point is that the amount of resources is infinite. In this way the user is able to calculate the amount of resources that is specifically needed to complete all the orders for this product within the desired time and with the chosen structure.

The user defines both the products and the production structure and therewith the level of detail of the model. Based on the goals of the study, the user decides which production steps are distinguished and on what level, in order to meet the desired structure, which processes the product undergoes, the sequence of the steps and which equipment, tools and materials are needed to execute these process steps for each product. This way a complete factory can be seen as a black box or one can define every detailed subtask.

Insight in the financial consequences of the designed production structure and corresponding order quantities is created in the Cost Estimating module. In addition to the parameters already set to the resources, tools and material in the Base module, this module adds all relevant financial parameters to the entities of the production structure. This is one of the most important elements for evaluating the robustness and financial risks of business cases.

The Goods Flow Control (GFC) module allows the user to analyze the effects of the designed goods flow control, the control over the order flow through the production structure. In addition to the base module that works with a simple release of orders and the assumption of infinite resource capacities, this module introduces decision rules for goods flow control. As a result not only capacity requirements can be evaluated, but also stock heights, Work In Process (WIP) and their associated cost parameters.

The main focus of Supply Chain Management (SCM) module lies with the external processes, the supply and demand of the production structure. The intention of the module is to create insight in the effects of material supply issues, such as order quantities, quality of the received goods and supplier lead times. With the SCM module the user can also aggregate the single production network to a higher level (black box) and so evaluate the supply chain as a network of several production units in order to determine the optimal requirements for the single production structure.

The Material Handling module is an add-on to the Goods Flow Control module. In the Goods Flow Control module several decision rules and supporting structure entities (e.g. stock points) are included in the design. The level of detail can be further increased by adding issues about physical layout of the site (locations of processes, resources, stock locations and work buffers) and the material handling processes between these locations to the model. With this module it is possible to evaluate how physical elements and restrictions in the production structure work out on both static and dynamic aspects. The static analysis comprises the occupancy of physical space in working buffers or stock points for materials. The dynamic analysis is focused on moving products through the system, for instance internal transport by forklift trucks.

\subsubsection{Input Settings}

The input of the model will be provided by a database application and concerns both the parameter settings for each model object and the information about the complete process structure itself, a processroute per product. Static data in the Base module is used to automatically generate this base production structure in the simulation model, by inserting the correct associating objects and linking them together. For capacity analysis the user can set the amount of orders per product that will be released per time interval. By creating more sequential time intervals and corresponding order quantities the user is able to define a production life cycle over the time horizon with increasing or decreasing production rates.

The financial parameters that can be set to the resources (personnel, equipment and materials) include fixed costs (e.g. investment, installation, residual value) and variable costs (e.g. costs of use, hourly tariffs). For materials and end-product these parameters include unit prices and residual values. By setting the parameters one defines the constant and variable costs per entity. 


\section{Hermelijn. Steenbakkers and Van Der Weij}

The influence of the financial environment on the total cost of the production structure is not negligible, so additional cost aspect that are taken into account include depreciation, inflation or deflation, interest, taxes, exchange rates, cost allocation and indirect costs. Depending to the selected modules, other financial settings will be available, for example Goods Flow Control requires availability of the stock holding cost. As the production of Fokker Aerostructures is characterized by long term manufacturing periods covering several years, time dependency plays a serious role in the cost. Therefore all settings can be varied per time-interval.

Each of the additional modules enables an additional group of input settings. The GFC-module expands the production structure with order management, stock points and working buffers to create elements to control the goods flow. Additional input concerns a selection of pre-defined well-known order management strategies and decision rules regarding resource, equipment and material selection and planning such as ConWIP, KanBan, MRP, Just-In-Time, several stock replenishment policies and mixed strategies of push and pull systems. The user can define to what extent the (different) rules are applicable to either single products and processes or groups.

When the Supply Chain Management module is switched on, the base model assumption that supplied materials are always available is overruled by a user-defined rule about material supply by the external suppliers. Per product (group) parameters like lead-time, order quantities and availability represent the supplier behavior. Another topic in the Supply Chain Management module is the demand rate. In this module the demand for every product can be defined on a more detailed level with parameters such as Stochastic inter-arrival times, demand quantities, seasonal influences and demand growth or decrease over time.

More complex is the input for the material handling module. Automation of input about resource allocation and planning is included, but it is hard to describe the complete layout in a database. For that purpose pre-defined building blocks in the application allow the user to design a layout, including location for machines, tools and working spaces, to create the production environment by drag and drop. By exchanging information between the model and the input database additional information can be provided about physical aspects such as width, length and height of the entities, but also the buffer sizes.

For moving products, materials and resources between the defined locations, a user can select different material handling resources like fork-lift trucks, conveyors, AGV's, robots or humans with their corresponding properties such as speeds, capacities and time schedules. The possible routes are defined through the combination of process steps for the products and locations.

\subsubsection{Output Parameters}

The performance of the value chain model can be represented by many indicators. Some of them are shown in the model, but all are exported to an external spreadsheet for possible further elaboration. These output parameters concern capacity related indicators like resource utilization, total production time per end product, but also financial indicators like fixed cost, variable cost, revenue, profit, return on investment and cash flow, that will help the user in evaluating the financial consequences of the designed production structure and support review of business cases. The results can be shown per product, process, resource or business unit, but also per time interval as the production and environment are subject to many dynamic features.

Goods Flow Control indicators are added to offer the user extra results, once the GFC module is selected. Performance indicators Work in progress, lead times, delivery dates and performance, stock amounts and stock availability allow evaluation of the designed goods flow control.

With the SCM module performance indicators concerning the externally supplied materials (stock figures, availability or demand acceptance) become available and the material handling module provides output about utilization and availability of physical areas, material handling equipment and travel times.

For all output parameters it is the user who defines the grouping and the level of detail of these characteristics. 


\section{VALUE CHAIN SIMULATION APPLICATION}

Based on the conceptualization, the application building has started module by module. In the first phase the base module (product and production structure) was developed together with the cost estimating module. This combination represents the basis of the solution which can be used to evaluate the consequence of product and process developments at the R\&D department for business cases.

For the technical implementation the simulation software Enterprise Dynamics and an input tool based on Microsoft Excel are applied. In the simulation environment several building object are created which represent the processes, the products, the resources, the materials, the orders and some support object. These object contain all the attributes and functionality to represent every product and production structure in aviation industry on every desired level of detail.

The model structure is such that the processes per Bill Of Material level are displayed above each other and the sequence of processes within a BOM level will be displayed from left to right. This provides a clear overview for the user. The interrelationship between objects are also visible in the model, as shown in Figure 3, which provides an overview of the representation of a structure.

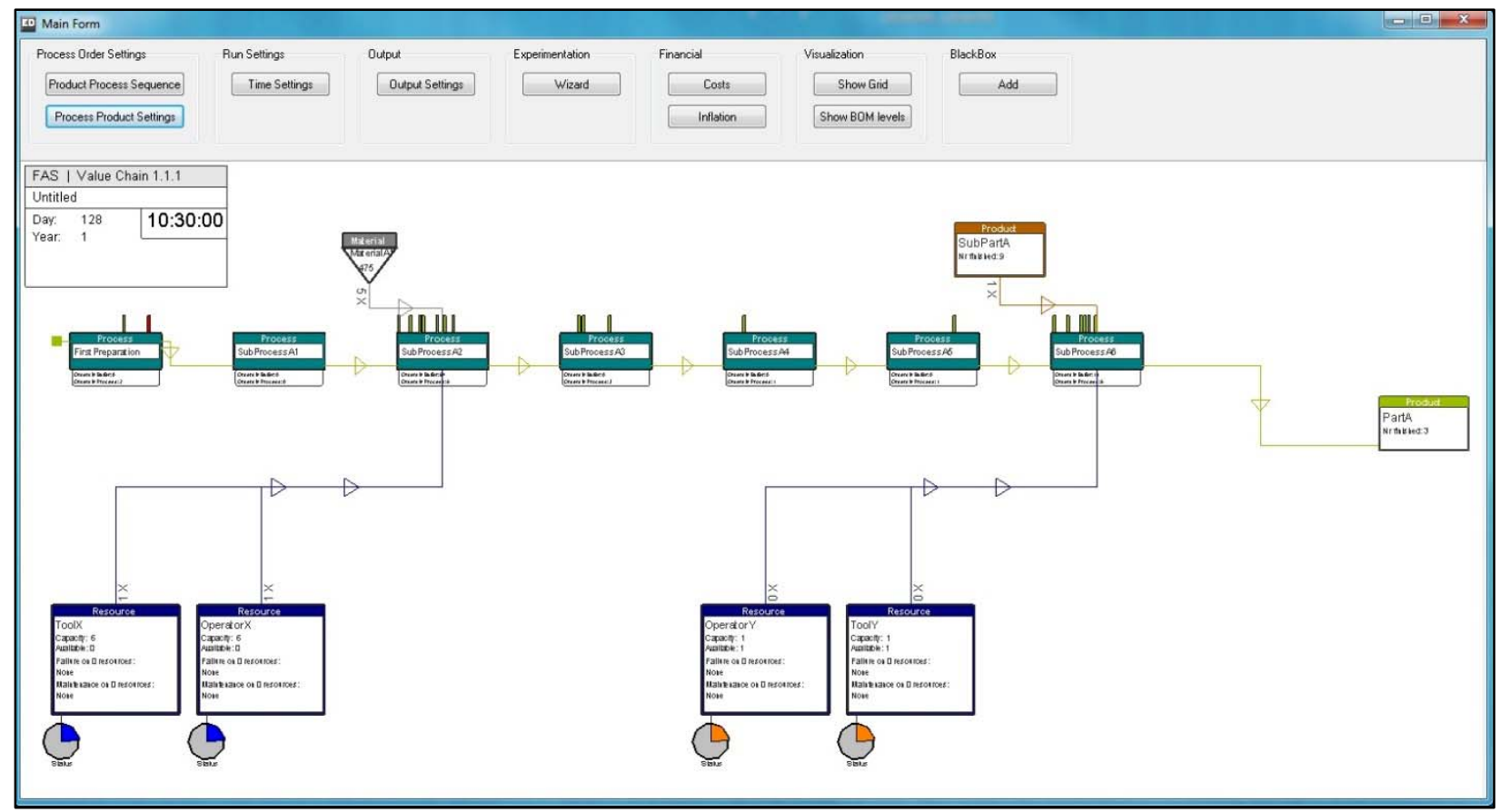

Figure 3: Visual overview of the main form of a model

A custom build user interface provides access to all kinds of tabular data, including simulation run set-up data, experimentation settings, financial data, output tables and the settings that describe the product and production structure. The application offers two different options for set-up and modification of these structures:

- Manual model building, by drag and drop functionality.

- Automatic model building, based on a structure input sheet;

Manual model building can be applied to create a simulation of a relatively small system or a system of which no structure data is available. Also for small model changes, this first option is the most convenient. The user selects the object from the available library and places the new elements by drag and drop into the model. After naming the element its settings can be used for structuring the model. 


\section{Hermelijn. Steenbakkers and Van Der Weij}

The first stage is to create the relation between materials and processes. In an easy graphical manner one can for every (sub) product or material select which processes are used and in which order to create a new (sub) product, see Figure 4. In such a way any structure, from simple to very complex and on any level of detail can be set up.

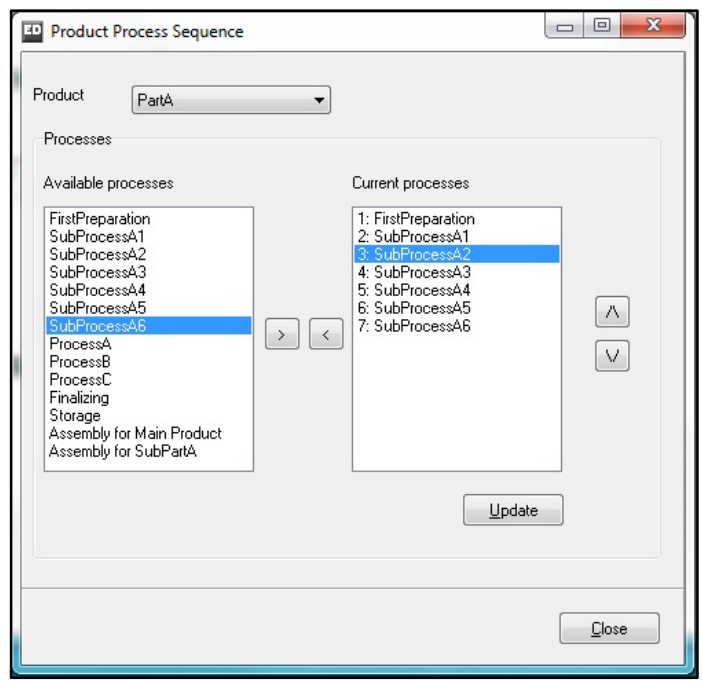

Figure 4: Product Process Sequence to define the process steps per product

Once the relation between products and processes is defined, the second stage is to allocate materials and resources to the processes. Per product-process combination it is possible to select one or more required resources, the amount of materials needed for this process and several specific settings like process times, scrap rates or learning curve. These settings can have great consequences on the performance of a system, but they are often omitted (or just roughly approximated) in regular cost calculating methods The GUI for this second stage is presented in Figure 5.

When the relations between all relevant model elements are defined the final stage in model set-up is defining the generic data. The element settings consists of (amongst others) availability and financial data. In all stages the user interfaces ensure that the correct model object are automatically connected to each other and filled with the appropriate data.

Besides manual model building there is an opportunity for fully Automatic model building in this Value Chain Simulator application. This option uses a structured input worksheet in Excel and is particularly useful for setting up large scale models or in case the model is subject to many modifications. The user has to complete a Microsoft Excel file consisting of several structured worksheets. This structure consists of the description of connections/relations between model elements, i.e. which processes are used (and in what order) to produce one product. Furthermore, for each of the processes the used materials and/or resources are defined. The content for these worksheets is directly imported from the BAAN ERPsystem at Fokker Aerostructures.

The complete structure can be imported and results in automatic build up of the model, including insertion of the corresponding model objects and allocation settings as in the manual mode. After the structure import, the model elements are also ordered based on the structure automatically.

The GUI's in the model can now be used to change specific properties when desired, but it is also possible to export the model content to Excel again, perform the modifications here and import the structure to the simulator once again. 


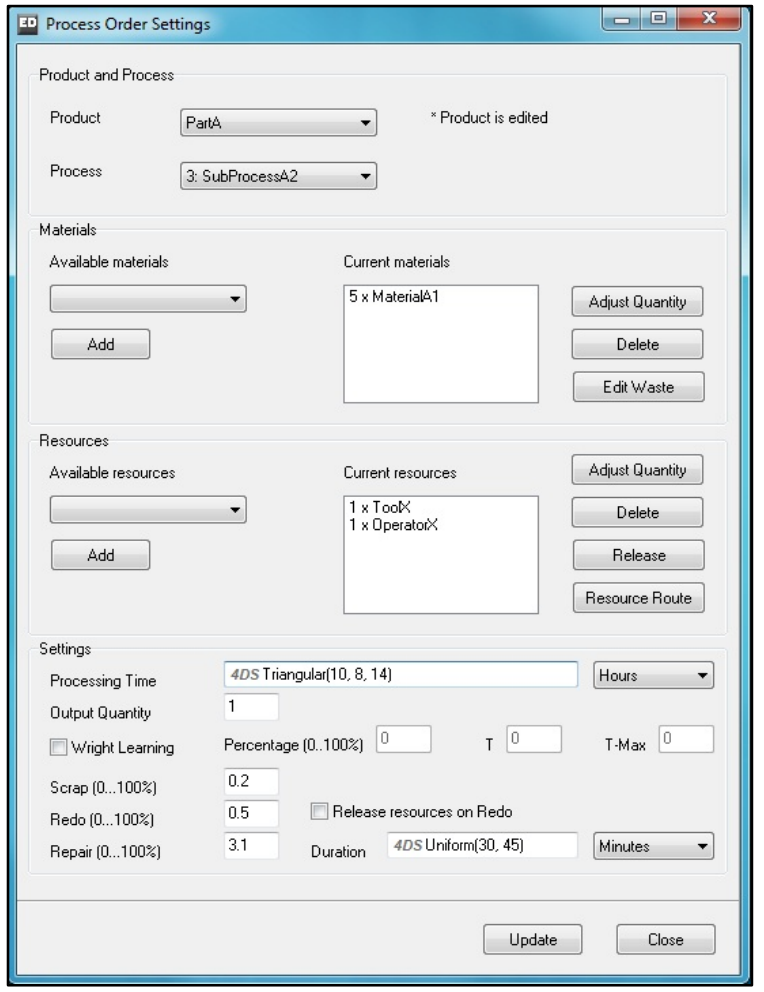

Figure 5: Settings for a product at a process

As soon as all settings are provided and validated by the user, experimentation can start. Replication length, number of replications and confidence interval boundaries are provided in the tool. The results are stored in tables and after all replications have finished, the complete output is exported automatically to an Excel output application. All relevant model output (consisting of both financial and logistic key performance indicators) is depicted in several graphs, see Figure 6. Furthermore raw output data can be viewed to evaluate other data of particular interest and create comparisons with other scenarios.

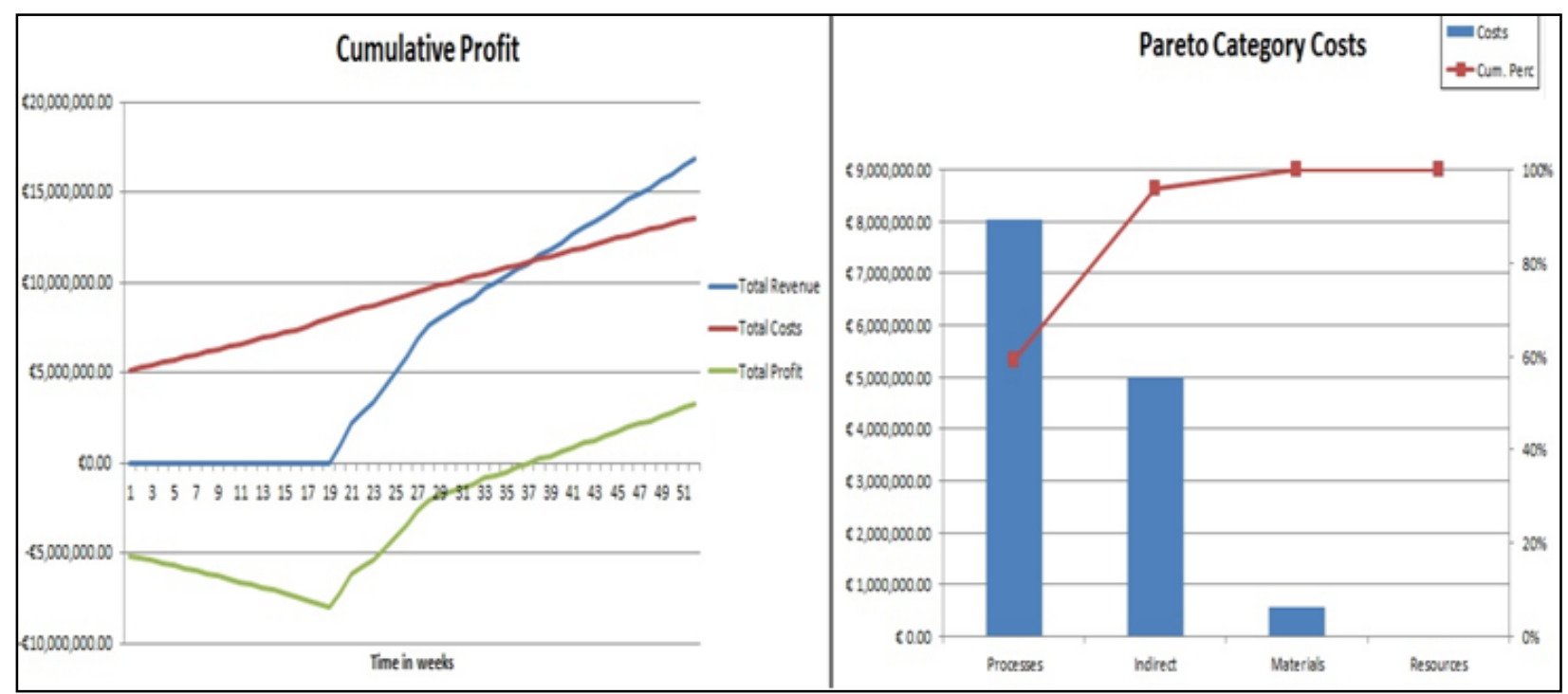

Figure 6: Cumulative profit over time and costs per category 


\section{RESULTS}

The purpose of the application is to provide Fokker Aerostructures insight in all activities involved in realizing their aviation products and the associated costs. The modular approach has so far resulted in an application that can be used for scenario comparison during the Research \& Development stages. The capacity analysis demonstrates the required capacity of resources, tools and other materials when producing a predefined set of orders and the financial consequences of the designed production structure and order quantities.

The following quote from Fokker Aerostructures R\&D engineering, expresses the degree to which the application meets the objectives set:

"When I was presented with the first version, I started using the application and became more and more enthusiastic about its possibilities. The application provides insight in numerous areas of the production process. The coupling with several financial aspects results in the immediate formation of a cost overview. The application offers the possibility to plan future production, providing us with insight in the future requirements concerning the resources and expenditures. In our R\&D department we use this tool to quantify the various technology choices."

At the same time some parts and concepts of the application have been used in business projects to improve the supply chain design and setting up an assembly line by creating models and conducting numerous experiments.

\section{FUTURE STEPS}

In the next phase INCONTROL will complete development of the other modules according to the design in the conceptualization phase. This will expand the opportunities for other departments at Fokker to benefit also from the application.

A final phase will be the development of the Total Quality management module. The quality is stated as the extent to which the product complies with the functional requirements stated by internal rules, legislation or strict customer requirement. There are many factors that influence this quality, like process design, material quality and resource quality. Defining quality is therefore a complex task and 'Total Cost of Ownership' is stated as the most ambitious goal. This module become relevant as soon as all other modules have been completely elaborated.

In parallel, additional work will be done for further integration of the simulation application with the ERP system, so that all relevant data can be exchanged unrestricted between ERP and the Fokker Value Chain Simulator.

\section{REFERENCES}

Porter, M.E. 1985. Competitive Advantage: Creating and Sustaining Superior Performance. Free Press, New York,

Steenbakkers, A.J. and S.W. van der Weij. 2010. Concept Functional Design, INCONTROL Simulation Solutions.

The TAPAS project. "The Thermoplastic Affordable Primary Aircraft Structure Consortium" 2011. http://www.tapasproject.nl 


\section{AUTHOR BIOGRAPHIES}

MARVIN HERMELIJN studied Operations Research at the Faculty of Economics and Business at the University of Amsterdam. Since 2007 he is working at INCONTROL Simulation Solutions. As Senior Simulation Engineer his focus is on developing simulation applications for industrial and logistics companies. His email address is Marvin.Hermelijn@incontrolsim.com.

JEROEN STEENBAKKERS is technical sales manager at INCONTROL Simulation Solutions, after having worked as simulation engineer for several years. He received a diploma in Industrial Engineering from the Technical University of Eindhoven. He can be contacted through the email address Jeroen.Steenbakkers@incontrolsim.com.

SIMON VAN DER WEIJ is team manager at INCONTROL Simulation Solutions. He is responsible for all simulation activities in the fields Industry, Logistics and Events. He graduated at the University of Twente, department of Mechanical Engineering in 1995 and has been working in the area of logistics simulation ever since. His email address is Simon.van.der.weij@incontrolsim.com. 